\title{
Use of Lung Ultrasound for Assessment of Lung Recruitment Maneuvers in Patients with ARDS
}

\author{
Waheed A. Radwan, Mohamed M. Khaled, Ayman G. Salman, Mohmed A. Fakher, Shady Khatab*(D) \\ Department of Critical Care, Faculty of Medicine, Cairo University, Cairo, Egypt
}

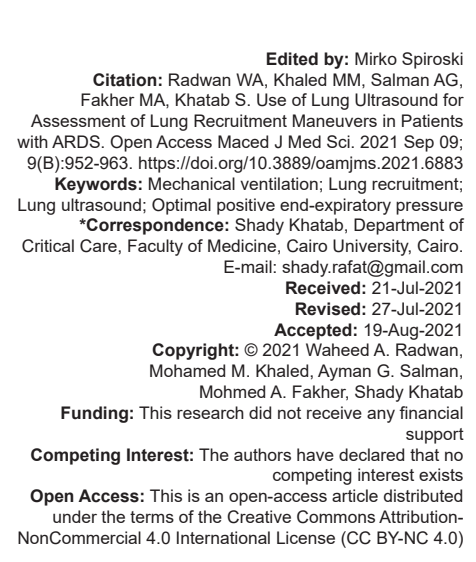

\section{Introduction}

Knowing the positive end-expiratory pressure (PEEP) helps in the treatment of acute respiratory distress syndrome (ARDS), which is associated with a significant risk of mortality [1], [2].

Methods used to evaluate the effect of PEEPinduced pulmonary recruitment include computed tomography $(C T)$, the static pressure-volume $(P-V)$ curve, and the oxygenation method [3], [4], [5].

However, the uses of these methods are limited due to objective factors. For example, for lung CT examination, critically ill patients must be transported out of the intensive care unit (ICU), which carries risk of transfer, high cost, and radiation exposure [6], [7], [8].

Static $\mathrm{P}-\mathrm{V}$ curve tracing requires deep sedation and muscle relaxation. The oxygenation method is the most commonly used in clinics, but it is necessary to repeat arterial blood collection many times, which is cumbersome and expensive. An ultrasonic examination is noninvasive, convenient, and reproducible. Due to the low water content, normal lung tissue cannot be detected by an ultrasonic examination; however, abnormal lung tissue has obvious changes in the gas/water ratio, which can be observed by the ultrasonic method. Studies have been evolving the value of ultrasound in the evaluation of lung recruitment [9], [10]. 


\section{Aim of the work}

The aim of this study was to explore the clinical value of ultrasonic monitoring in the assessment of pulmonary recruitment and the best PEEP.

\section{Patients and Methods}

This study was conducted as a non-randomized interventional prospective study on 65 patients between February 2017 and April 2019 in multicenter.

\section{Inclusion criteria}

Mechanically ventilated patients with ARDS with confirmed lung collapse were included in the study.

\section{Exclusion criteria}

The following criteria were excluded from the study:

- $\quad$ Mechanically ventilated patients not suffering from lung collapse and not needing lung recruitment maneuvers (RM)

- Mechanically ventilated patients who are hemodynamically unstable.

\section{Study groups} two groups

This study included 65 patients divided into

\section{Group A}

Included 50 mechanically ventilated, who underwent lung recruitment using lung ultrasound.

\section{Group B}

Included 15 mechanically ventilated patients who underwent lung recruitment using oxygenation index.

\section{ARDS in our study was identified using Berlin definition [11]}

1. Acute onset over 1 week or less

2. bilateral opacities consistent with pulmonary edema must be present and may be detected on CT or chest radiograph

3. $\mathrm{PF}$ ratio $<300 \mathrm{mmHg}$ with a minimum of $5 \mathrm{cmH} 20$ PEEP (or CPAP)

4. "Must not be fully explained by cardiac failure or fluid overload," in the physician's best estimation using available information - an "objective assessment" (e.g. echocardiogram) should be performed in most cases if there is no clear cause such as trauma or sepsis.

\section{All studied patients were subjected to}

Clinical assessment

- History taking (age, DM, hypertension, COPD, and steroid use)

Physical examination: Include general examination (including blood pressure, heart rate, temperature and respiratory rate) and local examination.

\section{Laboratory assessment}

$\mathrm{CBC}$, kidney function tests (serum creatinine and serum urea), and serum electrolyte (serum sodium and potassium) and arterial blood gases.

\section{Imaging}

- $\quad$ Chest X-ray

Using 4 quadrant score.

- $\quad$ Echocardiography

We assessed IVC diameter and RV functions using TAPSE.

\section{- $\quad$ Lung ultrasound}

We used lung ultrasound to determine the need for RM in each patient answering is whether our patient needs a RM. Ultrasound can detect the presence of lung collapse predominantly in dependent (dorsal) pulmonary areas following the gravity vector. Lung collapse can manifest as slight to moderated loss of lung aeration (from isolated to coalescent B-lines) up to complete atelectasis (sub-pleural consolidations with static air-bronchograms).

\section{Preparation}

Correction of hypovolemia and keeping mean arterial pressure (MAP) more than $75 \mathrm{mmHG}$ using IV fluids and inotropes if needed

- $\quad$ Adjustment of respiratory rate to $35 \mathrm{rpm}$ for 20 min before recruitment

- $\quad$ Sedation and neuromuscular blockade

- $\quad$ Keeping patient in supine or prone position

- $\quad$ Aspiration of lower airways secretions

- Increasing FIO2 to 1.0

- Increase airway pressure limit to $60 \mathrm{cmH} 2 \mathrm{O}$ and PEEP alarm limit to $40 \mathrm{~cm} \mathrm{H}_{2} \mathrm{O}$

Decreases trigger sensitivity

Change to pressure control ventilation. set pressure to $20 \mathrm{~cm} \mathrm{H} 2 \mathrm{O}$ above PEEP, I: E 1:1, RR 15

Then start recruitment. 


\section{Lung recruitment}

We divided patients two groups.

\section{Group A}

First, standard lung ultrasound scan was performed using different probes whose selection will depend on patient's age and body constitution as well as the clinical scenario. After performing a standard lung ultrasound scan, the selected probe is placed in the most dependent zone of the atelectatic lung. The RM maneuver is started searching the pressure level at which the consolidation-pattern disappears and progressive lung re-aeration is observed. Re-aeration usually follows a sequential pattern: Condensation - B2 lines - B1 lines to normal lung images but, sometimes, the pattern of condensation is transformed directly in a normal pattern. Then, the probe is placed in the same position in the contralateral lung and if a normal aeration is confirmed, this level of airway pressure is defined as the lung's opening pressure. After lung recruitment, it is necessary to perform a step-wise decrement in PEEP (PEEP trial) to detect the lung's closing pressure. The very moment when normal lung aeration images transform into a B1B2 pattern and consolidation pattern will define the closing pressure. The final selected level of PEEP is chosen to be $2 \mathrm{~cm} \mathrm{H} 2 \mathrm{O}$ above the detected closing pressure. Once the patient's opening and closing pressures are known, a new $\mathrm{RM}$ is performed reaching these target pressures and the identified PEEP level is set for ongoing ventilation.

\section{Group B}

We increase PEEP to $25 \mathrm{~cm} \mathrm{H} 2 \mathrm{O}$ for 10 breaths then decrease back to $20 \mathrm{~cm} \mathrm{H} 2 \mathrm{O}$ then increase PEEP to $30 \mathrm{~cm} \mathrm{H} 2 \mathrm{O}$ for 10 breaths then decrease back to $20 \mathrm{~cm} \mathrm{H} 2 \mathrm{O}$ then increase to $36 \mathrm{~cm} \mathrm{H} 2 \mathrm{O}$ in some patients and $38 \mathrm{~cm} \mathrm{H} 2 \mathrm{O}$ in others for 10 breaths then back to 20 $\mathrm{cmH} 2 \mathrm{O}$ then decrease PEEP in steps $1 \mathrm{~cm} \mathrm{H} 2 \mathrm{O}$ until $\mathrm{PaO} 2$ starts to fall. Then, we repeated recruitment and set PEEP $2 \mathrm{~cm} \mathrm{H} 2 \mathrm{O}$ above previously noted value.

\section{After lung recruitment}

- $\quad$ Reset alarm limits and reset I; E ratio to 1:2

- We assessed effect of lung recruitment on heart rate and mean arterial blood pressures (MAP)

- We measured P/F ratio immediately after recruitment and $12 \mathrm{~h}$ after recruitment

- We assessed compliance immediately after recruitment

- We assessed RV function by TAPSE using echocardiography

- We calculate ultrasound reaeration score in Group A (Table 1). (score more than 9 means that recruitment is good).
We did chest X-ray again 2 days after recruitment using 4 quadrant score.

\section{Ethical considerations}

Informed consent for participation in the study was obtained according to the guidelines of the institutional review boards for human subjects at the participating study centers.

Table 1: Ultrasound reaeration score

\begin{tabular}{lll}
\hline 1 point & 3 points & 5 points \\
\hline B1 $>\mathrm{N}$ & $\mathrm{B} 2>\mathrm{N}$ & $\mathrm{C}>\mathrm{N}$ \\
B2 $>\mathrm{B} 1$ & $\mathrm{C}>\mathrm{B} 1$ & \\
C $>\mathrm{B} 2$ & & \\
\hline
\end{tabular}

B1: Multiple well-defined either regularly spaced 7-mm apart or irregularly spaced B lines (moderate loss of lung aeration); B2: Multiple coalescent B lines (severe loss of lung aeration); C: Lung consolidation; N: Normal pattern (normal lung aeration). *The ultrasound reaeration score was calculated as follows: In a first step, ultrasound lung aeration (N, B1, B2, and C) was assessed in each of the 12 lung regions examined before and after application of PEEP. In a second step, ultrasound lung reaeration score was calculated as the sum of each score characterizing each lung region examined according to the scale shown in the table. Increasing aeration score less than 8 means bad ineffective recruitment and further trials in future table. Increasing a

\section{Statistical analysis}

Data were coded and entered using the statistical package SPSS version 25. Data were summarized using mean, standard deviation, median, minimum and maximum in quantitative variables and frequencies (number of cases) and relative frequencies (percentages) for categorical variables. Comparisons between groups were done using unpaired t-test in normally distributed quantitative variables while non-parametric Mann-Whitney test was used for non-normally distributed quantitative variables. For comparison of serial measurements within each patient paired, t test was used. For comparing categorical data, Chi-square $(\chi 2)$ test was performed. Exact test was used instead when the expected frequency is less than 5. $p<0.05$ was considered as statistically significant.

\section{Results}

This study was conducted on 120 patients, 30 were excluded as in whom lung collapse cannot be confirmed then the rest were 90 patients from whom another 25 patients excluded as they were hemodynamically unstable the rest 65 patients were divided into two groups:

- Group A: Included 50 mechanically ventilated patients with ARDS, underwent lung recruitment using lung ultrasound

Group B: Included 15 mechanically ventilated patients with ARDS, underwent lung recruitment using oxygenation index.

This Prospective study was held at many critical care departments around Egypt. 


\section{Description of all studied patients}

Our study included 65 patients whose were 35 male $(53.8 \%)$ and 30 females $(46.2 \%)$ with mean age $52.74 \pm 21.23$ with mean of APACHE II $18.63 \pm 6.58$.

24 patients from studied population were diabetic while 41 were not diabetic and 21 were hypertensive while 44 were not hypertensive and 9 were COPD and 56 were not COPD.

28 patients from studied patients were mechanically ventilated due to pneumonia, eight patients due to massive blood transfusion after severe bleeding, eight patients due to sepsis, five patients due to RTA, five patients due to aspiration of gastric contents, three patients due to organophosphorus poisoning, three patients due to acute pancreatitis, two patients due to smoke inhalation, two patients due to postoperative, and one patient due to near drowning.

\section{Demographic data of the studied population}

(Tables 2 and 3) patients with ARDS, hemodynamically stable, with confirmed lung collapse of whom 26 were males and 24 were females with mean age $(52.4 \pm 21.35)$

- $\quad$ Group B: Included 15 mechanically ventilated patients with ARDS, hemodynamically stable with confirmed lung collapse of whom nine were males and six were females with mean age $(53.87 \pm 21.51)$.

There was no statistically significant difference between Groups A and B regarding demographic data.

Table 2: Gender of studied populations

\begin{tabular}{|c|c|c|c|c|c|}
\hline & \multicolumn{4}{|c|}{ Method of lung recruitment } & \multirow[t]{3}{*}{$p$-value } \\
\hline & \multicolumn{2}{|c|}{ Group A } & \multicolumn{2}{|c|}{ Group B } & \\
\hline & Count & $\%$ & Count & $\%$ & \\
\hline \multicolumn{6}{|l|}{ Gender } \\
\hline Male & 26 & 52.0 & 9 & 60.0 & 0.586 \\
\hline Female & 24 & 48.0 & 6 & 40.0 & \\
\hline
\end{tabular}

(Tables 4 and 5)

\section{Clinical data of the studied population}

\section{Group A}

Regarding medical history, 20 patients were diabetic, 15 patients were hypertensive, and eight patient were COPD, with mean APACHE (18.16 \pm 6.36).

\section{Group B}

Regarding medical history, four patients were diabetic, six patient were hypertensive, and one patient was COPD, with mean APACHE (20.20 \pm 7.30$)$.

The both group were compared that revealed a no statistically significant difference between group as regard associated comorbid conditions, nor admission APACHEII score.

\section{Laboratory data (Table 6)}

Group A

The mean $\mathrm{HB}$ was $10.46 \pm 2$, the mean WBCs was $18650 \pm 7553$, the mean platelets 188300 \pm 92805 , the median of serum creatinine 1.6 with minimum 0.9 and maximum 11.8 , the mean of serum $\mathrm{Na}$ was $135.24 \pm 5$, and the mean of serum $\mathrm{K}$ was 4.4 \pm 0.79 .

\section{Group B}

The mean of HB was $10.79 \pm 2.4$, the mean of WBCs was $21066.67 \pm 9587.69$, the mean of platelets was $156200 \pm 57701.45$, the mean of serum creatinine was $1.93 \pm 0.58$, the mean of serum $\mathrm{Na}$ was $136.07 \pm$ 5.3 , and the mean of serum $\mathrm{K}$ was $4.68 \pm 0.74$.

There was no statistically significant difference between both groups as regard admission laboratory data.

\section{Mechanical ventilation (Table 7)}

\section{Group A}

Regarding cause of MV (21 patients) $42 \%$ was pneumonia, (6 patients) $12 \%$ was sepsis, (6 patients) $12 \%$ was severe bleeding with massive blood transfusion, $8 \%$ (4 patients) RTA, 8\% (4 patients) aspiration of gastric contents, and $4 \%$ (2 patients) for every one of the following, smoke inhalation, organophosphorus poisoning, acute pancreatitis, and post-operative and $2 \%$ (one patient) near drowning.

Regarding mode of ventilation $68 \%$ of patients was on volume controlled ventilation (VCV) and $32 \%$ were on pressure controlled ventilation (PCV) before lung recruitment.

Regarding steroid thereby $72 \%$ of patients had steroid therapy.

Table 3: Age of studied populations

\begin{tabular}{|c|c|c|c|c|c|c|c|c|c|c|c|}
\hline & Methoc & ig recru & & & & & & & & & $\mathrm{p}$-value \\
\hline & Group & & & & & Group & & & & & \\
\hline & Mean & SD & Median & Minimum & Maximum & Mean & SD & Median & Minimum & Maximum & \\
\hline Age (years) & 52.40 & 21.35 & 59.00 & 16.00 & 89.00 & 53.87 & 21.51 & 63.00 & 19.00 & 78.00 & 0.564 \\
\hline
\end{tabular}


Table 4: Comorbid conditions of studied populations

\begin{tabular}{|c|c|c|c|c|c|}
\hline & \multicolumn{4}{|c|}{ method of lung recruitment } & \multirow[t]{3}{*}{$p$-value } \\
\hline & \multicolumn{2}{|c|}{ Group A } & \multicolumn{2}{|c|}{ Group B } & \\
\hline & Count & $\%$ & Count & $\%$ & \\
\hline \multicolumn{6}{|l|}{ DM } \\
\hline Yes & 20 & 40.0 & 4 & 26.7 & \multirow[t]{2}{*}{0.348} \\
\hline No & 30 & 60.0 & 11 & 73.3 & \\
\hline \multicolumn{6}{|l|}{ HTN } \\
\hline Yes & 15 & 30.0 & 6 & 40.0 & \multirow[t]{2}{*}{0.535} \\
\hline No & 35 & 70.0 & 9 & 60.0 & \\
\hline \multicolumn{6}{|l|}{ COPD } \\
\hline Yes & 8 & 16.0 & 1 & 6.7 & \multirow[t]{2}{*}{0.672} \\
\hline No & 42 & 84.0 & 14 & 93.3 & \\
\hline
\end{tabular}

\section{Group B}

Regarding cause of MV $46.7 \%$ (7 patients) was pneumonia, $13.3 \%$ (2 patients) was sepsis, $13.3 \%$ (2 patients) was severe bleeding with massive blood transfusion, $6.7 \%$ (1 patient) for every one of following RTA, aspiration of gastric contents, organophosphorus poisoning, and acute pancreatitis.

Regarding mode of ventilation $66.7 \%$ of patients was on VCV and $33.3 \%$ was on PCV before lung recruitment.

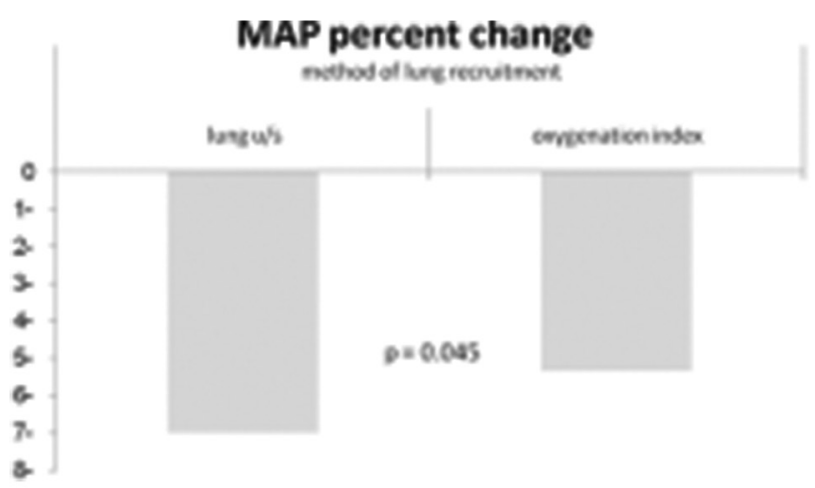

Figure 1: MAP percent change

Regarding steroid thereby $80 \%$ of patients had steroid thereby.

There was no statistically significant difference between both groups as regard causes of MV and mode of ventilation and steroid thereby.

Outcome of lung recruitment (Tables 8-10 and Figures 1 and 2)

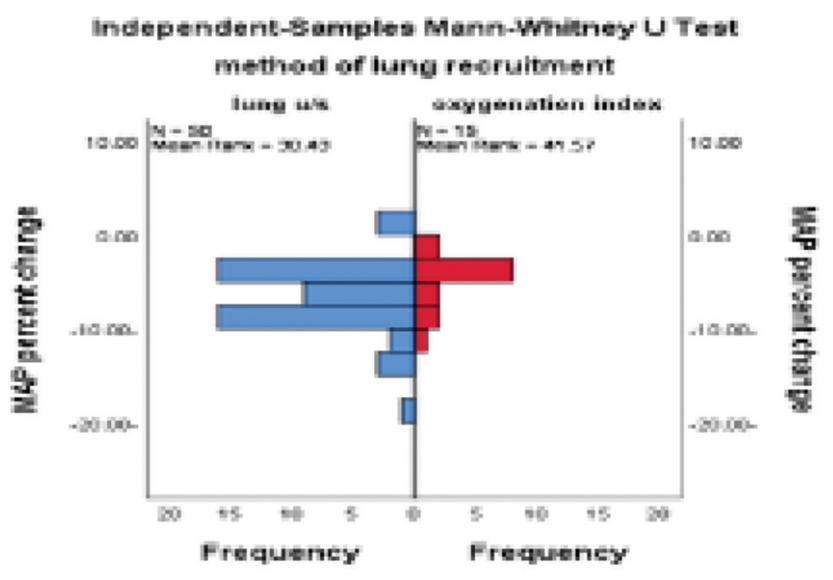

Figure 2: Independent-Samples Mann-Whitney test method of lung recruitment

Hemodynamics

\section{Group A}

The mean of heart rate before lung recruitment was $114.58 \pm 14.03$ and the mean of heart rate during lung recruitment was $124.52 \pm 13.58$ with statistically significant increase in heart rate during lung recruitment.

The mean of MAP was $100.02 \pm 6.26$ before lung recruitment and the mean of MAP during lung recruitment was $92.98 \pm 6.17$ with statistically significant decrease in MAP during lung recruitment.

\section{Group B}

The mean of heart rate before lung recruitment was $114.6 \pm 7.47$ and the mean of heart rate during lung recruitment was $121.87 \pm 6.89$ with statistically significant increase in heart rate during lung recruitment.

The mean of MAP was $99 \pm 3.95$ before lung recruitment and the mean of MAP during lung recruitment was $93.67 \pm 3.37$ with statistically significant decrease in MAP during lung recruitment.

There was statistically significant difference between both groups as regard MAP percent change before and during lung recruitment. In lung ultrasound group, the median of MAP percent change was -6.67 with maximum 0.00 and minimum -18.18 while in oxygenation index group the median of MAP percent

Table 5: APACHE score of studied populations

\begin{tabular}{|c|c|c|c|c|c|c|c|c|c|c|c|}
\hline & Metho & g recr & & & & & & & & & $\mathrm{p}$-value \\
\hline & Group & & & & & Group & & & & & \\
\hline & Mean & SD & Median & Minimum & Maximum & Mean & SD & Median & Minimum & Maximum & \\
\hline APACHE & 18.16 & 6.36 & 17.00 & 10.00 & 36.00 & 20.20 & 7.30 & 19.00 & 12.00 & 33.00 & 0.296 \\
\hline
\end{tabular}

Table 6: Admission laboratory data of studied populations

\begin{tabular}{|c|c|c|c|c|c|c|c|c|c|c|c|}
\hline & \multicolumn{10}{|c|}{ Method of lung recruitment } & \multirow[t]{3}{*}{ p-value } \\
\hline & \multicolumn{5}{|c|}{ Group A } & \multicolumn{5}{|l|}{ Group B } & \\
\hline & Mean & SD & Median & Minimum & Maximum & Mean & SD & Median & Minimum & Maximum & \\
\hline $\mathrm{HB}$ & 10.46 & 2.00 & 10.40 & 7.00 & 15.00 & 10.79 & 2.46 & 11.00 & 5.50 & 15.00 & 0.606 \\
\hline WBCs & 18650.00 & 7553.38 & 18000.00 & 3000.00 & 34000.00 & 21066.67 & 9587.69 & 21000.00 & 3000.00 & 41000.00 & 0.312 \\
\hline Platelets & 188300.00 & 92805.54 & 177000.00 & 50000.00 & 550000.00 & 156200.00 & 57701.45 & 167000.00 & 76000.00 & 256000.00 & 0.211 \\
\hline $\mathrm{Na}$ & 135.24 & 5.05 & 134.00 & 126.00 & 145.00 & 136.07 & 5.30 & 136.00 & 129.00 & 146.00 & 0.584 \\
\hline $\mathrm{K}$ & 4.40 & 0.79 & 4.25 & 3.20 & 6.70 & 4.68 & 0.74 & 4.90 & 3.60 & 5.90 & 0.221 \\
\hline creat & 1.90 & 1.55 & 1.60 & 0.90 & 11.80 & 1.93 & 0.58 & 1.80 & 1.30 & 3.30 & 0.151 \\
\hline
\end{tabular}


change was -4.72 with maximum -2.08 and minimum -12.83 with ( $p$-value 0.045).

\section{$P / F$ ratio immediate and $12 h$ after lung}

\section{recruitment (Tables 11-13)}

Table 7: Mechanical ventilation (cause, mode, and steroid therapy)

\begin{tabular}{|c|c|c|c|c|c|}
\hline & \multicolumn{4}{|c|}{ Method of lung recruitment } & \multirow[t]{3}{*}{$p$-value } \\
\hline & \multicolumn{2}{|c|}{ Group A } & \multicolumn{2}{|c|}{ Group B } & \\
\hline & Count & $\%$ & Count & $\%$ & \\
\hline \multicolumn{6}{|l|}{ Cause of ventilation } \\
\hline Pneumonia & 21 & 42.0 & 7 & 46.7 & 1 \\
\hline RTA & 4 & 8.0 & 1 & 6.7 & \\
\hline Severe bleeding with massive blood transfusion & 6 & 12.0 & 2 & 13.3 & \\
\hline Sepsis & 6 & 12.0 & 2 & 13.3 & \\
\hline Smoke inhalation & 2 & 4.0 & 0 & 0.0 & \\
\hline Organophosphorus poisoning & 2 & 4.0 & 1 & 6.7 & \\
\hline Acute pancreatitis & 2 & 4.0 & 1 & 6.7 & \\
\hline Aspiration of gastric contents & 4 & 8.0 & 1 & 6.7 & \\
\hline Near drowning & 1 & 2.0 & 0 & 0.0 & \\
\hline Post-operative & 2 & 4.0 & 0 & 0.0 & \\
\hline \multicolumn{6}{|l|}{ Mode of vent } \\
\hline VCV & 34 & 68.0 & 10 & 66.7 & 1 \\
\hline PCV & 16 & 32.0 & 5 & 33.3 & \\
\hline \multicolumn{6}{|l|}{ Steroids } \\
\hline Yes & 36 & 72.0 & 12 & 80.0 & 0.741 \\
\hline No & 14 & 28.0 & 3 & 20.0 & \\
\hline
\end{tabular}

\section{Group A}

The mean of Pao2/Fio2 ratio (PFR) before lung recruitment was $212.38 \pm 61.90$ and the mean of PFR immediate after lung recruitment was $398.36 \pm 66.24$ and the mean of PFR $12 \mathrm{~h}$ after lung recruitment was $345.32 \pm 71.72$ with statistically significant increase in both PFR immediate and $12 \mathrm{~h}$ after recruitment.

\section{Group B}

The mean of Pao2/Fio2 ratio (PFR) before lung recruitment was $218.78 \pm 36.37$ and the mean of PFR immediate after lung recruitment was $366.18 \pm 53.21$ and the mean of PFR $12 \mathrm{~h}$ after lung recruitment was $300.13 \pm 55.34$ with statistically significant increase in both PFR immediate and $12 \mathrm{~h}$ after recruitment.

There was statistically significant difference between both groups as regard PFR percent change before and immediate after lung recruitment. In lung ultrasound group, the mean of PFR percent change was $98.07 \pm 46.19$ while in oxygenation index group the

Table 8: Hemodynamic percent change regarding method of lung recruitment

\begin{tabular}{|c|c|c|c|c|c|c|c|c|c|c|c|}
\hline & \multicolumn{10}{|c|}{ Method of lung recruitment } & \multirow[t]{3}{*}{$p$-value } \\
\hline & \multicolumn{5}{|c|}{ Group A } & \multicolumn{5}{|c|}{ Group B } & \\
\hline & Mean & SD & Median & Minimum & Maximum & Mean & SD & Median & Minimum & Maximum & \\
\hline Pulse percent change & 9.05 & 5.97 & 7.47 & 0.00 & 29.69 & 6.42 & 2.86 & 5.45 & 3.82 & 14.02 & 0.066 \\
\hline MAP percent change & -6.99 & 3.61 & -6.67 & -18.18 & 0.00 & -5.34 & 2.67 & -4.72 & -12.38 & -2.08 & 0.045 \\
\hline
\end{tabular}

Table 9: Hemodynamics before and after recruitment in lung US group

\begin{tabular}{|c|c|c|c|c|c|c|c|c|c|c|c|}
\hline \multirow[t]{2}{*}{ Lung u/s } & \multicolumn{5}{|c|}{ Before recruitment } & \multicolumn{5}{|c|}{ After recruitment } & \multirow[t]{2}{*}{$\mathrm{p}$-value } \\
\hline & Mean & SD & Median & Minimum & Maximum & Mean & SD & Median & Minimum & Maximum & \\
\hline Pulse & 114.58 & 14.03 & 115.00 & 64.00 & 140.00 & 124.52 & 13.58 & 124.50 & 83.00 & 150.00 & $<0.001$ \\
\hline MAP & 100.02 & 6.26 & 100.00 & 85.00 & 110.00 & 92.98 & 6.17 & 92.50 & 82.00 & 105.00 & $<0.001$ \\
\hline
\end{tabular}

Table 10: Hemodynamics before and after recruitment in oxygenation index group

\begin{tabular}{|c|c|c|c|c|c|c|c|c|c|c|c|}
\hline \multirow[t]{2}{*}{ Oxygenation index } & \multicolumn{5}{|c|}{ before recruitment } & \multicolumn{5}{|c|}{ After recruitment } & \multirow[t]{2}{*}{$p$-value } \\
\hline & Mean & SD & Median & Minimum & Maximum & Mean & SD & Median & Minimum & Maximum & \\
\hline Pulse & 114.60 & 7.47 & 114.00 & 105.00 & 131.00 & 121.87 & 6.89 & 122.00 & 111.00 & 136.00 & $<0.001$ \\
\hline MAP & 99.00 & 3.95 & 99.00 & 94.00 & 106.00 & 93.67 & 3.37 & 93.00 & 90.00 & 101.00 & $<0.001$ \\
\hline
\end{tabular}

Table 11: PFR before recruitment and immediate and $12 \mathrm{~h}$ after recruitment in lung US group

\begin{tabular}{|c|c|c|c|c|c|c|c|c|c|c|c|}
\hline \multirow{2}{*}{ Lung u/s } & \multicolumn{5}{|c|}{ Before recruitment } & \multicolumn{5}{|c|}{ After recruitment } & \multirow[t]{2}{*}{$\mathrm{p}$-value } \\
\hline & Mean & SD & Median & Minimum & Maximum & Mean & SD & Median & Minimum & Maximum & \\
\hline PFR & 212.38 & 61.90 & 221.50 & 82.00 & 299.00 & 396.38 & 66.24 & 401.00 & 233.00 & 490.00 & $<0.001$ \\
\hline PFR $12 \mathrm{~h}$ & 212.38 & 61.90 & 221.50 & 82.00 & 299.00 & 345.32 & 71.72 & 337.00 & 149.00 & 456.00 & $<0.001$ \\
\hline
\end{tabular}

Table 12: PFR before recruitment and immediate and $12 \mathrm{hrs}$ after recruitment in oxygenation index group

\begin{tabular}{|c|c|c|c|c|c|c|c|c|c|c|c|}
\hline \multirow[t]{2}{*}{ Oxygenation index } & \multicolumn{5}{|c|}{ Before recruitment } & \multicolumn{5}{|c|}{ After recruitment } & \multirow[t]{2}{*}{$p$-value } \\
\hline & Mean & SD & Median & Minimum & Maximum & Mean & SD & Median & Minimum & Maximum & \\
\hline PFR & 218.73 & 36.37 & 222.00 & 150.00 & 284.00 & 366.13 & 53.21 & 391.00 & 276.00 & 432.00 & $<0.001$ \\
\hline PFR $12 \mathrm{~h}$ & 218.73 & 36.37 & 222.00 & 150.00 & 284.00 & 300.13 & 55.34 & 300.00 & 212.00 & 400.00 & $<0.001$ \\
\hline
\end{tabular}

Table 13: PFR percent change and PFR after $12 \mathrm{~h}$ percent change regarding method of recruitment

\begin{tabular}{|c|c|c|c|c|c|c|c|c|c|c|c|}
\hline & \multicolumn{10}{|c|}{ Method of lung recruitment } & \multirow[t]{3}{*}{$\mathrm{p}$-value } \\
\hline & \multicolumn{5}{|c|}{ Group A } & \multicolumn{5}{|c|}{ Group B } & \\
\hline & Mean & SD & Median & Minimum & Maximum & Mean & SD & Median & Minimum & Maximum & \\
\hline PFR percent change & 98.07 & 46.19 & 76.71 & 49.44 & 240.91 & 68.57 & 15.90 & 63.02 & 47.89 & 101.01 & 0.009 \\
\hline PFR after $12 \mathrm{~h}$ percent change & 69.95 & 33.02 & 61.44 & 16.54 & 177.27 & 37.85 & 18.31 & 32.83 & 14.59 & 93.47 & $<0.001$ \\
\hline
\end{tabular}


mean of PFR percent change was $68.57 \pm 15.90$ with $(p=0.009)$.

There was statistically significant difference between both groups as regard PFR percent change before and $12 \mathrm{~h}$ after lung recruitment. In lung ultrasound group, the mean of PFR after 12 hrs percent change was $69.95 \pm 33.02$ while in oxygenation index group the mean of PFR after $12 \mathrm{~h}$ percent change was $37.85 \pm$ 18.31 with $(p<0.001)$.

\section{Compliance (Tables 14-16) \\ Group A}

The mean of compliance before lung recruitment was $30.53 \pm 2.72$ and the mean of compliance after lung recruitment was $41.14 \pm 4.94$ with statistically significant increase in compliance after recruitment.

\section{Group B}

The mean of compliance before lung recruitment was $30.68 \pm 3.13$ and the mean of compliance after lung recruitment was $39.33 \pm 3.85$ with statistically significant increase in compliance after recruitment.

There was no statistically significant difference between both groups as regard compliance percent change before and after lung recruitment.

\section{Right ventricular function using (TAPSE),} (Tables 17-19 and Figure 3)

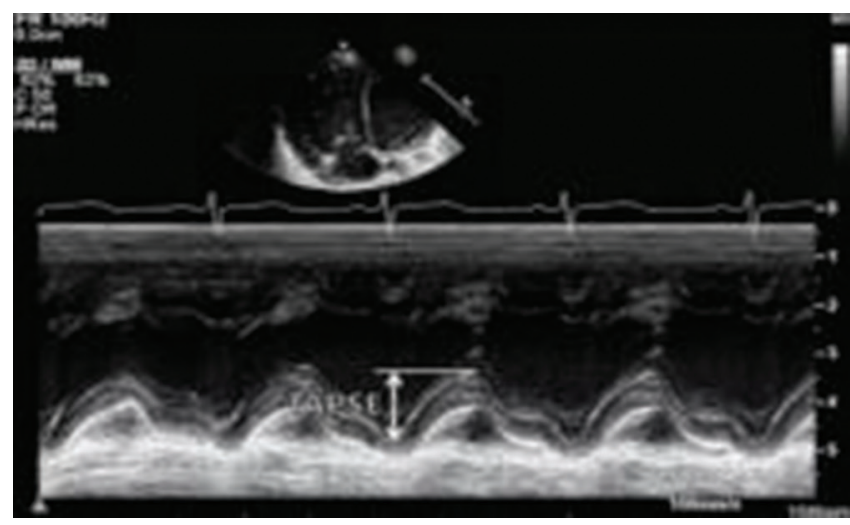

Figure 3: Right ventricular function using TAPSE

Table 14: Compliance before and after recruitment in lung US group

\begin{tabular}{|c|c|c|c|c|c|c|c|c|c|c|c|}
\hline \multirow[t]{2}{*}{ Lung $\mathrm{u} / \mathrm{s}$} & \multicolumn{5}{|c|}{ Before recruitment } & \multicolumn{5}{|c|}{ After recruitment } & \multirow[t]{2}{*}{$p$-value } \\
\hline & Mean & SD & Median & Minimum & Maximum & Mean & SD & Median & Minimum & Maximum & \\
\hline Compliance & 30.68 & 3.13 & 31.00 & 22.00 & 36.00 & 41.14 & 4.94 & 43.00 & 27.00 & 48.00 & $<0.001$ \\
\hline
\end{tabular}

Table 15: Compliance before and after recruitment in oxygenation index group

\begin{tabular}{|c|c|c|c|c|c|c|c|c|c|c|c|}
\hline \multirow[t]{2}{*}{ Oxygenation index } & \multicolumn{5}{|c|}{ Before recruitment } & \multicolumn{5}{|c|}{ After recruitment } & \multirow[t]{2}{*}{$p$-value } \\
\hline & Mean & SD & Median & Minimum & Maximum & Mean & SD & Median & Minimum & Maximum & \\
\hline Compliance & 30.53 & 2.72 & 31.00 & 26.00 & 36.00 & 39.33 & 3.85 & 40.00 & 32.00 & 44.00 & $<0.001$ \\
\hline
\end{tabular}

Table 16: Compliance percent change before and after recruitment regarding method

\begin{tabular}{|c|c|c|c|c|c|c|c|c|c|c|c|}
\hline & \multicolumn{10}{|c|}{ Method of lung recruitment } & \multirow[t]{3}{*}{$\mathrm{p}$-value } \\
\hline & \multicolumn{5}{|c|}{ Group A } & \multicolumn{5}{|c|}{ Group B } & \\
\hline & Mean & SD & Median & Minimum & Maximum & Mean & SD & Median & Minimum & Maximum & \\
\hline Compliance percent change & 34.35 & 12.62 & 34.38 & 12.50 & 62.96 & 28.90 & 7.54 & 29.03 & 17.24 & 44.83 & 0.145 \\
\hline
\end{tabular}

Table 17: RV function before and after recruitment in lung US group

\begin{tabular}{|c|c|c|c|c|c|c|c|c|c|c|c|}
\hline \multirow[t]{2}{*}{ Lung u/s } & \multicolumn{5}{|c|}{ Before recruitment } & \multicolumn{5}{|c|}{ After recruitment } & \multirow[t]{2}{*}{ p-value } \\
\hline & Mean & SD & Median & Minimum & Maximum & Mean & SD & Median & Minimum & Maximum & \\
\hline RV & 1.77 & 0.09 & 1.79 & 1.50 & 1.92 & 1.83 & 0.09 & 1.83 & 1.60 & 1.99 & $<0.001$ \\
\hline
\end{tabular}

Table 18: RV function before and after recruitment in oxygenation index group

\begin{tabular}{|c|c|c|c|c|c|c|c|c|c|c|c|}
\hline \multirow{2}{*}{ Oxygenation index } & \multicolumn{5}{|c|}{ Before recruitment } & \multicolumn{5}{|c|}{ After recruitment } & \multirow[t]{2}{*}{$p$-value } \\
\hline & Mean & SD & Median & Minimum & Maximum & Mean & SD & Median & Minimum & Maximum & \\
\hline $\mathrm{RV}$ & 1.72 & 0.04 & 1.72 & 1.67 & 1.80 & 1.78 & 0.05 & 1.79 & 1.69 & 1.89 & $<0.001$ \\
\hline
\end{tabular}

Table 19: RV function percent change before and after recruitment regarding method

\begin{tabular}{|c|c|c|c|c|c|c|c|c|c|c|c|}
\hline & \multicolumn{10}{|c|}{ Method of lung recruitment } & \multirow[t]{3}{*}{$p$-value } \\
\hline & \multicolumn{5}{|c|}{ Group A } & \multicolumn{5}{|c|}{ Group B } & \\
\hline & Mean & SD & Median & Minimum & Maximum & Mean & SD & Median & Minimum & Maximum & \\
\hline RV percent change & 3.32 & 2.32 & 2.93 & $-0.58-$ & 9.04 & 3.44 & 1.72 & 3.39 & 0.56 & 6.78 & 0.607 \\
\hline
\end{tabular}

Table 20: Opening pressure and optimal PEEP in both groups

\begin{tabular}{|c|c|c|c|c|c|c|c|c|c|c|c|}
\hline & \multicolumn{10}{|c|}{ Method of lung recruitment } & \multirow[t]{3}{*}{$p$-value } \\
\hline & \multicolumn{5}{|c|}{ Group A } & \multicolumn{5}{|c|}{ Group B } & \\
\hline & Mean & SD & Median & Minimum & Maximum & Mean & SD & Median & Minimum & Maximum & \\
\hline Opening pre & 37.28 & 1.25 & 38.00 & 35.00 & 40.00 & 36.67 & 0.98 & 36.00 & 36.00 & 38.00 & 0.085 \\
\hline Optimal PEEP & 14.64 & 1.08 & 15.00 & 11.00 & 16.00 & 13.13 & 0.74 & 13.00 & 12.00 & 14.00 & $<0.001$ \\
\hline
\end{tabular}


Table 21: Day of recruitment and length of MV in both methods

\begin{tabular}{|c|c|c|c|c|c|c|c|c|c|c|c|}
\hline & \multicolumn{10}{|c|}{ Method of lung recruitment } & \multirow[t]{3}{*}{$p$-value } \\
\hline & \multicolumn{5}{|c|}{ Group A } & \multicolumn{5}{|c|}{ Group B } & \\
\hline & Mean & SD & Median & Minimum & Maximum & Mean & SD & Median & Minimum & Maximum & \\
\hline Day of MV & 2.32 & 1.33 & 2.00 & 1.00 & 6.00 & 2.47 & 1.30 & 2.00 & 1.00 & 5.00 & 0.708 \\
\hline Day of recruitment & 4.62 & 1.63 & 5.00 & 2.00 & 8.00 & 5.00 & 1.65 & 4.00 & 3.00 & 8.00 & 0.432 \\
\hline Length of ventilation (days) & 9.60 & 2.71 & 9.50 & 5.00 & 16.00 & 11.27 & 4.57 & 10.00 & 7.00 & 25.00 & 0.236 \\
\hline
\end{tabular}

\section{Group A}

The mean of RV function using TAPSE before lung recruitment was $1.77 \pm 0.09$ and the mean of RV using TAPSE after lung recruitment was $1.83 \pm 0.09$ with statistically significant increase in $\mathrm{RV}$ function after recruitment.

\section{Group B}

The mean of RV function using TAPSE before lung recruitment was $1.72 \pm 0.04$ and the mean of RV function using TAPSE after lung recruitment was $1.78 \pm$ 0.05 with statistically significant increase in RV function after recruitment.

There was no statistically significant difference between both groups as regard RV function (using TAPSE) percent change before and after lung recruitment.

\section{(Table 20 and Figure 4)}

\section{optimal PEEP}

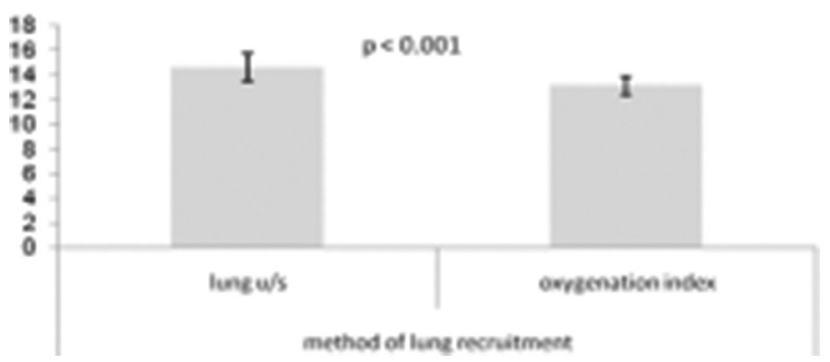

Figure 4: Optimal PEEP

\section{Group A}

The mean of opening pressure was $37.28 \pm$ 1.25 and the mean of optimal PEEP was $14.64 \pm 1.08$.

\section{Group B}

The mean of opening pressure was $36.67 \pm$ 0.98 and the mean of optimal PEEP was $13.13 \pm 0.74$.

There was statistically significant difference between both groups as regard optimal pressure with $p<0.001$

Day of recruitment and length of mechanical ventilation (Table 21)

\section{Group A}

The mean of day of recruitment was $4.62 \pm$ 1.63 and the mean of length of MV was $9.6 \pm 2.71$.

\section{Group B}

The mean of day of recruitment was 5.00 \pm 1.65 and the mean of length of MV was $11.27 \pm$ 4.57 .

There was no statistically significant difference between both groups as regard day of neither recruitment nor length of MV.

\section{Complications (Table 22)}

\section{Group A}

In lung US group, three patients out 50 developed complications in form of pneumothorax with $6 \%$ percent.

Table 22: Complication in both methods

\begin{tabular}{|c|c|c|c|c|c|}
\hline & \multicolumn{4}{|c|}{ Method of lung recruitment } & \multirow{3}{*}{ p-value } \\
\hline & \multicolumn{2}{|c|}{ Group A } & \multicolumn{2}{|c|}{ Group B } & \\
\hline & Count & $\%$ & Count & $\%$ & \\
\hline \multicolumn{6}{|c|}{ Complications } \\
\hline Yes & 3 & 6.0 & 2 & 13.3 & \multirow[t]{2}{*}{0.325} \\
\hline No & 47 & 94.0 & 13 & 86.7 & \\
\hline \multicolumn{6}{|c|}{ Mortality } \\
\hline Yes & 18 & 36.0 & 6 & 40.0 & \multirow[t]{2}{*}{0.778} \\
\hline No & 32 & 64.0 & 9 & 60.0 & \\
\hline
\end{tabular}

\section{Group B}

In oxygenation index group, two patients out 15 had complications in form of pneumothorax with $13.3 \%$ percent.

There was no statistically significant difference between both groups as regard complication.

\section{(Table 23)}

\section{Mortality before hospital discharge}

\section{Group A}

In lung US group, 18 patients out 50 died with percent 36\%.

Table 23: Mortality in both groups

\begin{tabular}{llllll}
\hline & \multicolumn{2}{l}{ Method of lung recruitment } & \multirow{2}{*}{ p-value } \\
\cline { 2 - 4 } & Group A & \multicolumn{3}{ll}{ Group B } & \\
\cline { 2 - 5 } & Count & $\%$ & Count & $\%$ & \multirow{2}{*}{0.778} \\
\hline Mortality & 18 & 36.0 & 6 & 40.0 & \\
Yes & 32 & 64.0 & 9 & 60.0 & \\
No & 32 & & & & \\
\hline
\end{tabular}




\section{Group B}

In oxygenation index group, six patients out 15 died with percent $40 \%$.

There was no statistically significant difference between both groups as regard complication.

\section{Discussion}

The possibility of exploring the lung using ultrasound, at the bedside and noninvasively, is gaining popularity among intensivists. Lung ultrasound would be of minor interest if the usual tools (bedside radiography, and CT) did not have drawbacks (irradiation, low information content for radiography, and need for transportation...). Our study showed that ultrasound can be used instead of CT in many cases. Lung ultrasound is part of critical ultrasound, defined as a whole-body approach using simple machines, one universal probe, and new applications [12].

As techniques become more refined and experience increases, the lung US pattern, which was considered "artifacts," has become an important means of evaluating pathophysiologic changes in the lungs [11].

When used dynamically, lung US is reported to be an effective means of assessing PEEP-induced pulmonary reaeration [13].

Our study used ultrasound scoring and the oxygenation method to monitor the endpoint of lung recruitment. After lung recruitment, with decreasing PEEP levels, recruited alveoli collapsed and the oxygenation level decreased. Until the endpoint, more alveoli collapsed and pulmonary parenchyma changes were aggravated, which led to dramatic changes in the air/water ratio that could be detected by ultrasound; these changes were manifested as replacing of the A line by the $B$ line or air bronchogram consolidation inside the original $\mathrm{B}$ line. So that during lung recruitment, intermittent high airway pressure was first given to open the collapsed alveoli fully; this was followed by applying appropriate pressure to maintain the opening of the alveoli [14].

Choosing an appropriate PEEP is the key to maintain the alveoli open after lung recruitment. However, one feature of segment lesions in ARDS is that the lesions of the lower and dorsal lungs are severe, while those of the upper and anterior lungs are slight. The best PEEP was considered when the trapped alveoli was just expanded and the alveolar gas quantity increased by more than $20 \%$; in addition, part of the shunt was removed, oxygenation was improved, lung injury induced by shear stress by repeated alveolar opening and closing was reduced, and pulmonary circulation was improved [15].
Considering the heterogeneous nature of morphologic changes in ARDS, it is important to establish whether lung US can show optimal changes in lung morphologic characteristics that correlate with maximal improvements in aeration. In our study, we found that the protocol for reaeration in US-guided lung recruitment was an effective means of guiding alveolar recruitment in ARDS compared with the maximal oxygenation-guided method.

The main clinical manifestations of ARDS are progressive respiratory failure and hypoxemia, which arise as a consequence of decreased lung aeration, heterogeneous alveolar collapse and consolidation, and a mismatch in ventilation and perfusion. The oxygenation index has consequently been adopted as an indicator of lung recruitment [16].

The maximal oxygenation method, based on multiple comparisons of arterial blood gas analysis, can also be influenced by a wide range of factors other than alveolar recruitment [17].

While lung US can image the entire lung tissue, the technique appears to have many of the advantages of CT for detecting dynamic changes in lung aeration, but it also is noninvasive, requires no exposure to $X$-rays, is straight forward, and can be performed at the bedside.

We found that lung US evaluation was a more effective means of assessing ARDS compared with the maximal oxygenation method, either for comprehensive assessment on ARDS development or searching for the recruitment end point.

First, the lung US method can describe the distribution of consolidation in an ARDS lung instead of simply indicate an oxygenation level.

Second, the significant improvement in arterial oxygenation during the 5-cm H2O PEEP trial was not consistent with the dynamic changes seen on US evaluations, which were limited to the anterior and medial lung regions.

Third, our findings have preliminary verified the protocol for reaeration in US-guided lung recruitment, which considered the lung US reaeration score as the end point and was more beneficial in guiding lung recruitment, thus highlighting the value of assessing the effectiveness of a lung recruitment strategy with lung US. Our investigation showed that the protocol for reaeration in US-guided lung recruitment, guided by the maximal reaeration score, achieved higher opening pressures and resulted in further improvements in lung recruitment compared with the maximal oxygenation method.

Last, lung US-guided recruitment improved aeration in all regions of the lung, whereas the results in the oxygenation group showed that lung heterogeneity did not improve completely, as indicated by the remaining consolidation and interstitial edema in the medial and posterior parts of the lung. On the other hand, to avoid potential hyperinflation and lung injury caused by increasing airway pressure constantly, the protocol for 
reaeration in US-guided lung recruitment proposes a systematic method for achieving an optimized pressure to balance potential harm and benefits.

In our study, we found that heart rate was significantly increased and MAP was significantly decreased immediately after lung recruitment compared with basal state, and that match with study done by Tang et al., between 2015 and 2017 on 40 patients with ARDS who divided randomly into two groups, group underwent lung recruitment using ultrasound (20 patients) and another group underwent recruitment using oxygenation index (20 patients) [18].

And also match study done by Grasso et al. on 22 patients with ARDS who were recruited from the ICUs of the Di Venere, Policlinico (University of Bari), and S. Chiara (University of Pisa) hospitals. Inclusion criteria were age more than 18 years and diagnosis of ARDS. Exclusion criteria were cardiogenic pulmonary edema (clinically suspected or pulmonary artery occlusion pressure more than $18 \mathrm{mmHg}$ ), history of ventricular fibrillation or tachyarrhythmia, unstable angina or myocardial infarction within the preceding month, preexisting chronic obstructive pulmonary disease, MAP less than $65 \mathrm{mmHg}$ (despite attempts to increase blood pressure with fluid and vasopressors, as clinically indicated), anatomic chest wall abnormalities, chest tube with persistent air leak, pregnancy, and intracranial abnormality [19].

In our study, there was statistically significant difference between both groups as regard MAP percent change before and during lung recruitment. In lung ultrasound group, MAP decrease more significantly than in oxygenation group and that mismatch study done by Tang et al. and our explanation is that in lung recruitment, we reareate previously derecruited alveoli through application of an intentional and transient increase in the transalveolar pressure leading to decrease of MAP and in our study we found that in lung ultrasound group we used higher opening pressure and also higher optimal PEEP leading to more increase in transalveolar pressure leading to more decrease in venous return leading to more decrease in MAP.

In our study, we found that lung recruitment in both groups significantly increased $\mathrm{Pao} 2 / \mathrm{Fio} 2$ ratio immediately after recruitment compared with basal state and also significantly increase dynamic compliance compared with basal state, and that match study done by Tang et al., study done by Grasso et al., and study done by Li et al. in which 12 rabbits with saline lavageinduced lung injury were randomly divided into two groups: One with alveolar recruitment guided by lung US and the other with alveolar recruitment guided by maximal oxygenation [18], [19], [20].

In our study also we found that the increase in PF ratio immediately was significantly more in ultrasound group than in oxygenation group that results match with study done by Tang et al. and also match with study done by Li et al., [18], [20].
In our study, we found also that $\mathrm{P} / \mathrm{F}$ ratio $12 \mathrm{~h}$ after recruitment decreased compared with $\mathrm{P} / \mathrm{F}$ ratio immediately after recruitment but significantly increased compared with basal state before recruitment and also we found that the increase in P/F ratio $12 \mathrm{~h}$ after recruitment was more significantly in lung ultrasound group than in oxygenation group and that can be explained, as in our study, we found our optimal PEEP using lung ultrasound that was $2 \mathrm{~cm} \mathrm{H} 2 \mathrm{O}$ above closing pressure and we applied that optimal PEEP after recruitment and so that alveoli kept opened and not collapsed again

In our study, we found that lung recruitment (both lung ultrasound and oxygenation group) significantly increase RV function using TAPSE compared with basal state and that match with study done by Longo et al. that performed on 40 anaesthetized patients with New York Heart Association class I or II, preoperative left ventricular ejection fraction at least $50 \%$ and Euro score 6 or less scheduled for cardiac surgery with cardiopulmonary bypass (CPB) and that study found that atelectasis after CPB impairs RV function but this can be resolved by lung recruitment using $10 \mathrm{cmH} 2 \mathrm{O}$ of PEEP [21].

In our study, we found that both opening pressure and optimal PEEP were significantly higher in lung ultrasound group than in oxygenation group and that matches study done by Tang et al. and also study done by Li et al., [18], [20].

In our study, opening pressure was $37.28 \pm$ 1.25 in lung ultrasound group and was $36.67 \pm 0.98$ and optimal PEEP was $14.64 \pm 1.08$ in lung ultrasound group and was $13.13 \pm 0.74$ in oxygenation group.

In our study, pneumothorax developed with percent $6 \%$ (three patients out of 50 ) in lung ultrasound group, and developed with percent $13.3 \%$ (two patients out 15 ) in oxygenation group with total percent $7 \%$ of all recruited patients of study (five patients out 65).

Mercat et al. had A multicenter randomized controlled trial of 767 adults with ALI conducted in 37 ICUs in France from September 2002 to December 2005 Comparing the effect on outcome of a strategy for setting PEEP aimed at increasing alveolar recruitment while limiting hyperinflation to one aimed at minimizing alveolar distension in patients with ALI. And that study showed pneumothorax with percent of $6.8 \%$ in recruited patients [22].

In our study, death before hospital discharge was with percent $36 \%$ (18 patients out of 50 ) in lung ultrasound group while was with percent $40 \%$ (six patients out of 15 ) in oxygenation group with overall mortality percent of all recruited patients in our study $36.9 \%$ (24 patients out 65) while in study done by Mercat et al., death before hospital discharge percent was 35.4\% [22].

Our study has certain limitations as is that study was single center study and we did not use CT chest to confirm lung collapse. 


\section{Conclusion}

Lung US is an effective mean of evaluating and guiding alveolar recruitment in ARDS. Compared with the maximal oxygenation-guided method, the protocol for reaeration in US-guided lung recruitment achieved a higher opening pressure, resulted in greater improvements in lung aeration, and substantially reduced lung heterogeneity in ARDS.

\section{Recommendations}

- $\quad$ Lung ultrasound may be used to detect the lung recruitment endpoint and guide the best PEEP, as that can improve lung compliance of ARDS patients and oxygenation effect while avoiding potential hyperinflation and lung injury caused by increasing airway pressure constantly Further study is needed to compare sensitivity and specificity of each CT chest and lung ultrasound in confirming lung collapse.

\section{References}

1. Heinrichs W. Positive end-expiratory pressure (PEEP). Anaesthesist. 1992;41(10):653-69.

PMid:1443517

2. Mortelliti MP, Manning HL. Acute respiratory distress syndrome. Indian Pediatr. 2010;47(10):861-8.

PMid:21048239

3. Tehrani FT. A closed-loop system for control of the fraction of inspired oxygen and the positive end-expiratory pressure in mechanical ventilation. Comput Biol Med. 2012;42(11):1150-6. https://doi.org/10.1016/j.compbiomed.2012.09.007 PMid:23058098

4. Ingaramo OA, Ngo T, Khemani RG, Newth CJ. Impact of positive endexpiratory pressure on cardiac index measured by ultrasound cardiac output monitor*. Pediatr Crit Care Med. 2014;15(1):1520. https://doi.org/10.1097/pcc.0b013e3182976251 PMid:24389709

5. Regli A, Chakera J, de Keulenaer BL, Roberts B, Noffsinger B, Singh $B$, et al. Matching positive endexpiratory pressure to intra-abdominal pressure prevents end-expiratory lung volume decline in a pig model of intra-abdominal hypertension. Crit Care Med. 2012;40(6):1879-86. https://doi.org/10.1097/ ccm.0b013e31824e0e80 PMid:22488004

6. Oks M, Cohen RI, Koenig SJ, Narasimhan M. The use of point of care ultrasound in the medical intensive care unit reduces healthcare cost and patient radiation exposure. Chest. 2013;144(4):542A. https://doi.org/10.1378/chest.1704776

7. Parker MS, Hui FK, Camacho MA, Chung JK, Broga DW, Sethi NN. Female breast radiation exposure during CT pulmonary angiography. AJR Am J Roentgenol. 2005;185(5):1228-33. https://doi.org/10.2214/ajr.04.0770

PMid:16247139

8. Xie Z, Liao X, Kang Y, Zhang J, Jia L. Radiation exposure to staff in intensive care unit with portable CT scanner. Biomed Res Int. 2016;2016:5656480. https://doi.org/10.1155/2016/5656480 PMid:27556036

9. Mohanty K, Blackwell J, Egan T, Muller M. Characterization of the lung parenchyma using ultrasound multiple scattering. Ultrasound Med Biol. 2016;140(5):3186-7. https://doi. org/10.1016/j.ultrasmedbio.2017.01.011 PMid:28318888

10. Skouras C, Davis ZA, Sharkey J, Parks RW, Garden OJ, Murchison JT, et al. Lung ultrasonography as a direct measure of evolving respiratory dysfunction and disease severity in patients with acute pancreatitis. HPB (Oxford). 2015;18(2):159-69. https://doi.org/10.1016/j.hpb.2015.10.002 PMid:26902135

11. Via G, Storti E, Gulati G, Neri L, Mojoli F, Braschi A. Lung ultrasound in the ICU: From diagnostic instrument to respiratory monitoring tool. Minerva Anestesiol. 2012;78(11):1282-96.

PMid:22858877

12. Foti G, Cereda M, Sparacino ME, de Marchi L, Villa F, Pesenti A Effects of periodic lung recruitment maneuvers on gas exchange and respiratory mechanics in mechanically ventilated acute respiratory distress syndrome (ARDS) patients. Intensive Care Med. 2000;26(5):501-7. https://doi.org/10.1007/s001340051196 PMid: 10923722

13. Bouhemad B, Brisson $H$, Le-Guen $M$, Arbelot C, Lu Q, Rouby JJ. Bedside ultrasound assessment of positive endexpiratory pressure-induced lung recruitment. Am J Respir Crit Care Med. 2011;183(3):341-7. https://doi.org/10.1164/ rccm.201003-0369oc PMid:20851923

14. Mannes GP, Boersma WG, Baur CH, Postmus PE. Adult respiratory distress syndrome (ARDS) due to bacteraemic pneumococcal pneumonia. Eur Respir J. 1991;4(4):503-4 PMid: 1855580

15. Jin WH, Jung H, Choi HS, Hong SB, Lim CM, Koh Y. Efficacy of positive end-expiratory pressure titration after the alveolar recruitment manoeuvre in patients with acute respiratory distress syndrome. Crit Care. 2009;13(1):R22. https://doi. org/10.1186/cc7725

PMid:19239703

16. Gattinoni L, Caironi P, Cressoni M, Chiumello D, Ranier VM, Quintel $\mathrm{M}$, et al. Lung recruitment in patients with the acute respiratory distress syndrome. $\mathrm{N}$ Engl J Med. 2006;354(17):1775-86. https://doi.org/10.1056/nejmoa052052 PMid:16641394

17. Tugrul S, Cakar N, Akinci O, Ozcan PE, Disci R, Esen F, et al. Time required for equilibration of arterial oxygen pressure after setting optimal positive end-expiratory pressure in acute respiratory distress syndrome. Crit Care Med. 2005;33(5):9951000. https://doi.org/10.1097/01.ccm.0000163402.29767.7b PMid: 15891327

18. Tang KQ, Yang SL, Zhang B, Liu HX, Ye DY, Zhang HZ, et al. Ultrasonic monitoring in the assessment of pulmonary recruitment and the best positive end-expiratory pressure. Medicine (Baltimore). 2017;96(39):e8168. https://doi. org $/ 10.1097 / \mathrm{md} .0000000000008168$ PMid:28953669

19. Grasso S, Mascia L, Del Turco M, Malacarne P, Giunta F, Brochard L, et al. Effects of recruiting maneuvers in patients with acute respiratory distress syndrome ventilated with protective ventilatory strategy. Anesthesiology. 2002;96(4):795802. https://doi.org/10.1097/00000542-200204000-00005 
PMid:11964585

20. Li DK, Liu DW, Long $Y$, Wang $X T$. Use of lung ultrasound to assess the efficacy of an alveolar recruitment maneuver in rabbits with acute respiratory distress syndrome. J Ultrasound Med. 2015;34(12):2209-15. https://doi.org/10.7863/ultra.14.11051 PMid:26543168

21. Longo S, Siri J, Acosta C, Palencia A, Echegaray A, Chiotti I, et al. Lung recruitment improves right ventricular performance after cardiopulmonary bypass: A randomised controlled trial.
Eur J Anaesthesiol. 2017;34(2):66-74. https://doi.org/10.1097/ eja.0000000000000559

\section{PMid:27861261}

22. Mercat A, Richard JM, Vielle B, Jaber S, Osman D, Diehl JL, et al. Positive end-expiratory pressure setting in adults with acute lung injury and acute respiratory distress syndrome: A randomized controlled trial. JAMA. 2008;299(6):646-55. https://doi.org/10.1001/jama.299.6.646

PMid:18270353 